\title{
QTL Mapping of Novel Genomic Regions for Yield Related Traits in Doubled Haploid (DH) Population Derived from the Popular Rice Hybrid KRH-2
}

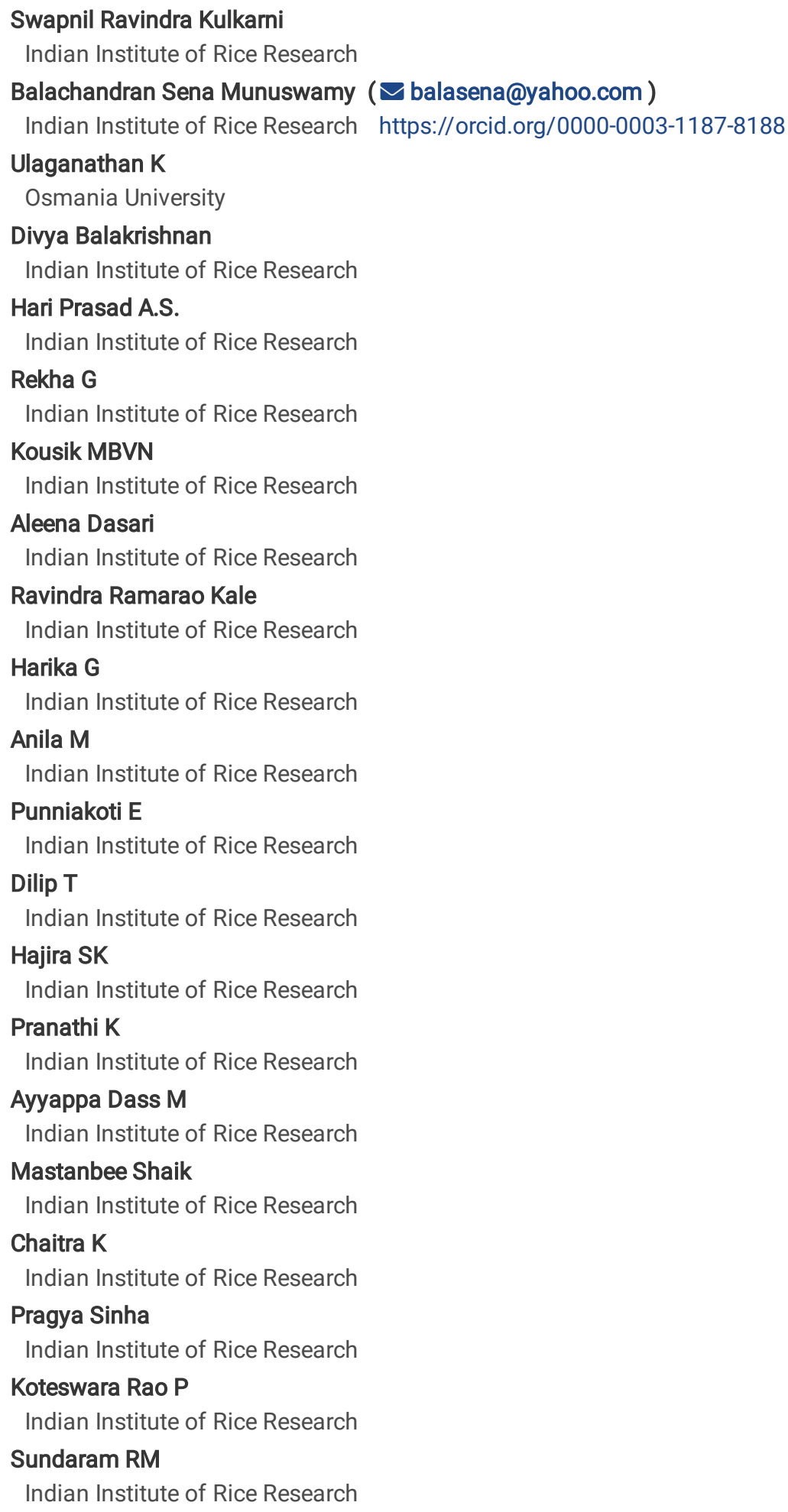


Keywords: Doubled Haploid Lines (DHLs), Androgenesis, KRH-2, QTL mapping, SSR markers, Inclusive Composite Interval Mapping (ICIM), QTL hotspots

Posted Date: September 28th, 2020

DOI: https://doi.org/10.21203/rs.3.rs-62169/v1

License: (c) (i) This work is licensed under a Creative Commons Attribution 4.0 International License. Read Full License 


\section{Abstract}

Background: Rice, being the principal food crop and major nutritional source for more than half of the global population, is also an important source of livelihood in many South and South-East Asian countries. Amidst diminishing natural resources and many biotic-abiotic stresses, increasing the yield of rice varieties remains a challenging task. Identification of novel and yield augmenting alleles from stable rice hybrids is crucial to facilitate their marker-assisted transfer into various genetic backgrounds.

Results: Quantitative trait loci (QTL) mapping using a population of 125 doubled haploid (DH) lines developed from the cross IR58025A/KMR3R and 126 polymorphic SSR; EST-derived SSR markers led to the identification of 12 each of major-minor effect QTLs for yield related traits. Major effect QTLs were detected for traits namely days to fifty percent flowering, test $(1,000)$ grain weight, plant height, panicle weight, panicle length, flag leaf width, flag leaf length, biomass and total grain yield/plant explaining the phenotypic variability in the range of 29.95\%-56.75\%. QTL hotspots were detected on chromosome 3 for the traits, panicle length and total grain yield/plant and on chromosome 6 for the traits, panicle length, flag leaf length and total grain yield/plant. Though many of these QTLs were noted to co-localize with the QTL regions reported in earlier studies, five novel and major effect QTLs for panicle length, biomass, flag leaf width, panicle weight, plant height and three novel minor effect QTLs for panicle weight and fertile grains per panicle, were identified in this study.

Conclusions: Through this study, both major-minor effect novel QTLs for crucial yield related traits, viz., fertile grains per panicle, panicle length, panicle weight were identified. Further, the QTL hotspots identified on two different chromosomes for flag leaf length, panicle length and total grain yield/plant shall not only help in understanding the underlying genetic mechanisms of yield regulation but also would provide an insight into the genetic synchrony among the various yield related traits in contributing for yield heterosis. The identified QTL hotspots after their validation can be deployed in breeding programs targeted towards improvement of yield heterosis.

\section{Background}

The global demand of rice, the major staple food crop in Asian countries, is expected to reach 852 million tons by 2035 [1]. Even though the present and future demand of rice is exceptionally high, during the last decade, its annual yield increment was observed to be less than or equal to $1 \%$ [2]. Therefore, it will be a daunting task to boost rice production in the coming years, amidst diminishing natural resources, increasing incidence of biotic and abiotic stresses and a rapidly changing climate. In such a challenging scenario, hybrid rice technology, which is based on exploitation of yield heterosis, offers hope for sustaining rice cultivation as hybrids have consistently demonstrated yield increment of minimum $10 \%-15 \%$ when compared with the best varieties [3]. Notwithstanding the release of, 105 rice hybrids for cultivation in India over the years, the hybrid rice area occupies a limited 3 million hectares, only $~ 6.8 \%$ of the total area of 44 million hectares under rice [4]. Certain technical challenges such as modest yield advantage, grain quality issues in the first and second generation rice hybrids, along with high-cost of the hybrid seeds has resulted in its lower adoption in Asian countries like India [5]. In order to make the technology popular in India, [6] opined that the yield advantage of these hybrids should be $20 \%-30 \%$ more than the popular and inbred varieties. This projected increment in the heterotic levels of rice hybrids is possible by improving the parental lines for crucial agro-morphological traits like yield and its related traits by employing biotechnological tools in conjunction with traditional breeding techniques [7]. One such approach is the exploitation of quantitative trait loci associated with total grain yield and its associated traits. To achieve this, it is imperative to deploy non-segregating populations, which show wide phenotypic variation for the key agro-morphological traits under study. Such non-segregating populations include doubled haploid lines (DHLs), near isogenic lines (NILs), recombinant inbred lines (RILs), chromosomal segment substitution lines (CSSLs). Among them the DHLs can be a very useful resource for identification of yield QTLs and heterosis associated loci $[8,9,10,11,12]$, in the context of hybrid rice improvement. Development of the above mentioned immortal and fixed populations from popular high-yielding rice hybrids could be a valuable resource for mapping various agronomically important traits relevant to hybrid rice [13, 14].

The process of molecular mapping and utilization of quantitative trait loci (QTL) is extensively employed for enhancing rice yields $[15,16,17$, 18]. This has been possible because a number of novel QTL loci those govern the expression of yield and its related traits were identified along with their precise chromosomal position and trait-enhancing effects $[19,20]$. Identification of such beneficial yield enhancing QTLs was possible with a combinatorial approach of molecular and conventional breeding. Marker assisted selection (MAS) an important strategy of molecular breeding led to the introgression of such novel yield related QTLs in low-yielding rice varieties which resulted in their higher and consistent yields [20,21,22]. Lower rice yield was attributed mainly to lesser number of productive tillers and limited number of filled grains/panicle [23]. Moreover, it was conspicuously noted that the loss of favorable genes governing the panicle length trait resulted in smaller panicles which in turn resulted in lower yields [24]. This problem was addressed by the marker assisted transfer of genes/QTLs associated with enhancing the total grain number into popular but low yielding cultivars [25]. Also, the utility of MAS and Hap 3 haplotypes [26, 27] for achieving higher rice yields in numerous rice species is well understood. MAS strategy played a crucial role not only in transferring yield related genes/QTLs, but also conferring tolerance to many abiotic stresses namely drought [28], cold tolerance [29] and several biotic stresses [30] in rice. Hence, understanding the mode of gene action of such beneficial genes/QTLs is crucial from the breeding point of view [31]. Apart from the genes/QTLs those influence the trait expression, the variegated effects of environmental interactions on the latter cannot be ruled out [32].

Page $3 / 20$ 
Therefore, only those QTLs which demonstrate consistent expression across various environments with lower predominance of genotype (G) $\times$ environment $(E)$ are important from plant breeding perspective [33, 34].

Genetic architecture of rice yield involves a study of yield related trait components which influence the former's expression. Moreover, understanding the various modes of gene actions which contribute to yield trait's expression is also a crucial part of genetic architecture. Out of the various agro-morphological traits that influence the genetic architecture of rice yield, two traits namely the number of productive tillers or tillering number (TN) and panicle morphology are known to have a higher influence [35] and which share a common developmental biology [36]. As explained by [36], apical growth and branching regulates the tiller number (TN) and panicle architecture. The first group of genes i.e. monoculm (MOC) genes [39], was observed to regulate the axillary bud formation, thus influencing the TN and yield traits. The second group of genes, the tillering dwarf genes, was identified to effectively regulate the tillering number $[41,42]$. The third group of genes was observed to influence the TN trait by regulating the plant hormonal pathway $[43,44,45]$. Other genetic mechanisms which regulate the TN are follows: Negative regulation of axillary bud growth [46], positive regulation of TN through enhanced photosynthesis [47]. Jiang et al. [48] identified four candidate genes which were assumed to have a role in influencing the variations in tiller numbers and are awaiting validation. Pleiotropic interactions two traits viz., between yield and days to fifty percent flowering were determined by [49] using a rice MAGIC population.

Though there are several reports on the development of immortal populations from elite rice hybrids for QTL mapping [50, 51], deployment of doubled haploid (DH) population developed from elite hybrids is limited [52,53]. Considering the above mentioned points, the present investigation was undertaken with the objectives of developing doubled haploid (DH) population from the cross IR58025A/KMR3R and identifying yield and its related QTLs from the population by employing hyper-variable SSR markers. Further, efforts were also made to identify the architecture of novel QTLs.

\section{Results}

\section{Statistical analysis of agro-morphological trait performance of DHLs}

The frequency distribution of the key agro-morphological traits indicates their normal distribution for three consecutive seasons (Figure 1). Significant phenotypic variation (Supplementary Table 1) was noted between the different traits in the DHL population. As shown in Supplementary Table 2, significant differences with respect to the traits, DFF, GP, TGW, PT, BM, FGP, PW, PL, FLL, FLW, PH and YLD, were observed among the individual entries of the DHL population through combined ANOVA. The statistical variation in agro-morphological data in the form of descriptive statistics table is presented in Supplementary Table 3. Genotypic correlation among the traits was observed to be very strong and positive than the phenotypic correlation. A significant and positive genotypic correlation among the traits was observed, particularly for the traits, YLD and GP $(r=0.02)$, TGW $(r=0.20)$, PW $(r=0.61)$, PT $(r=0.37), P L(r=0.81), B M(r=0.44)$ at $1 \%$ level of significance (Supplementary Table 4). At $5 \%$ level of significance, traits YLD and FGP were observed to be positively correlated $(r=0.04)$. A negative correlation was noted for the traits, YLD and DFF $(r=-0.11)$, FLL $(r=-0.06)$ at $5 \%$ level of significance. Moreover, very strong and positive genotypic correlation was also observed between the traits PL and GP ( $r=0.15)$, TGW $(r=0.24)$, PW $(r=0.59)$, PH $(r=0.22)$, FLW $(r=0.14)$, PT $(r=0.27)$ at $1 \%$ level of significance. Genetic variability estimates (Supplementary Table 5 ) demonstrated a very high level of genotypic variance $(\mathrm{Vg})$ and phenotypic variance $(\mathrm{Vp})$ for the traits viz., FGP (2750 and 3088, respectively), GP (2517 and 2950). Among all the traits, the lowest $\mathrm{Vg}$ and $\mathrm{Vp}$ values were observed for FLW trait $(0.02$ and 0.14$)$, respectively. Cumulatively for all the agro-morphological traits, the phenotypic variance was observed to be higher than the genotypic variance. Higher genotypic coefficient of variance (GCV) and phenotypic coefficient of variance $(\mathrm{PCV})(>20 \%)$ was observed for the traits PW, PL, BM and YLD among which the highest GCV-PCV value was observed for the trait BM (37.49 and 38.36, respectively). Moderate GCV-PCV values (between 10\%-20\%) was observed for the traits, GP, FGP, TGW, PH, FLL, FLW, PT. Low value (less than 10\%) of the GCV-PCV was observed for the trait DFF. Broad sense heritability $\left(H^{2}\right)$ was noted to be in the range of $16.80 \%$ (FLW trait) to $95.52 \%$ (BM trait). The highest genetic advance (GA) value was observed for the trait FGP (101.94) whereas the lowest value of 0.13 was associated with FLW trait. The genetic advance per mean (GAM) was observed in the range of 75.49 (BM trait) and 6.41 (DFF trait). The DH population demonstrated significant statistical variability for the traits under study; hence, the population can be considered suitable to be subjected to QTL mapping analysis [50].

\section{QTL identification with SSR markers}

Using a set of 126 hyper-variable SSR and EST derived SSR markers (Supplementary Table 6), a total of 12 major effect-12 minor effect QTLs were detected among the 125 DHLs for all the traits except for the total number of grains per panicle (GP) and number of productive tillers (PT) (Additional file 1; Table 1 and Supplementary Table 7).

As per [54], a QTL with greater than 20 PVE \% is considered to be having a major effect. Twelve major effect QTLs were detected for all the traits viz., days to fifty percent flowering (DFF), total grains per panicle (GP), test $(1,000)$ grain weight (TGW), productive tillers (PT), biomass 
(BM), fertile grains per panicle (FGP), panicle weight (PW), panicle length (PL), flag leaf length (FLL), flag leaf width (FLW), plant height (PH) and total grain yield/plant (YLD) on chromosomes 3, 4, 6, 7, 9 and 12. The LOD scores of these QTLs were observed to be in the range of 2.7016.51 with PVE\% between 29.95\%-56.75\%. By using 126 hyper-variable SSRs, 15,750 data-points were noted to be amplified. Out of them, 652 data-points to the tune of $4.13 \%$ did not amplify and therefore considered to be missing data points.

\section{Days to fifty percent flowering (DFF):}

A major effect QTL, qDFF12-1, was identified with a LOD score of 3.64 and PVE value of $48.60 \%$. This QTL with size 5.01 cM was flanked by the markers RM27966 (46.68 cM) and RM235 (51.69 cM). Among them, RM27966 was the closest and with the additive effect value of 9.77 and it was inherited from IR58025A (Additional file 1; Table 1, Figure 2). The cumulative effect (RSq value) of the major and minor effect QTLs is shown in Additional file 1; Table 1 and Supplementary Table 7, respectively.

\section{Total grain yield per plant (YLD):}

Two major effect QTLs, namely, qYLD3-1 and $q Y L D 6-1$, were identified with LOD scores of 16.51 and 14.36, respectively and the PVE\% of both these QTLs was 56.75\% and 35.29\%, respectively. The QTL, qYLD3-1, with a size of $19.71 \mathrm{cM}$ was flanked by the markers, RM448 (93.41 cM) and RM15679 (113.12 cM) and it was closely associated with RM15679. The QTL, qYLD6-1 of 15.15 cM was observed to be flanked by RM7023 (53.95 cM) and RM586 (69.10) with RM7023 being the closely associated marker. The negative additive effect value, -7.81, of $q Y L D 3-$ 1 indicated that the favorable allele was inherited from KMR-3R (Table 1, Figure 2, Supplementary Figure 1a-I). An additive effect value of 7.43 for qYLD6-1, showed that it was inherited from IR58025A (Additional file 1; Table 1, Figure 2, Supplementary Figure 1b-I). The cumulative phenotypic variance (RSq) explained by both the QTLs accounted up to $97.26 \%$ indicating that both are major effect in nature.

\section{Test $(1,000)$ grain weight (TGW):}

A total of two major QTLs namely, qTGW6-1 and qTGW7-1, were identified for this trait. The first major QTL, qTGW6-1 was observed to be flanked by RM19410 (25.11 cM) and RM19429 (27.50 cM) with RM19429 being the closest associated marker. The LOD score and PVE\% of the 2.39 cM sized QTL, qTGW6-1, was observed to be 4.19 and $46.78 \%$, respectively. The additive effect value of -2.11 for this QTL showed the inheritance of favorable allele from KMR-3R (Additional file 1; Table 1, Figure 2). qTGW7-1, was observed to be located in between the markers, RM20948 (167.52 cM) and RM21649 (172.53 cM) with RM21649 to be identified as closely associated marker. The LOD score and PVE\% of this QTL was observed to be 3.06 and $29.95 \%$, respectively. The size of this QTL being $5.01 \mathrm{cM}$, was identified to have a negative additive effect value of -1.24 demonstrating its inheritance from KMR-3R (Additional file 1; Table 1, Figure 2). The cumulative effect of both these QTLs on the phenotype accounted up to $72.94 \%$ (Additional file 1; Table 1).

\section{Panicle weight (PW):}

qPW9-1, a major effect QTL, was observed to be located between the flanking SSR markers namely, RM23959 (95.82 cM) and RM23958 (110.97 cM) with a LOD score of 3.44 and PVE\% of 33.19\%. Among the two markers, RM23958 was the closest associated with respect to this QTL. The additive effect of this $15.15 \mathrm{cM}$ sized QTL was -6.46 indicating its inheritance from KMR-3R. The cumulative phenotypic variance (RSq value) of major and minor effect QTLs identified for this trait was observed to be $46.42 \%$ (Additional file 1; Table 1, Figure 2, Supplementary Figure 1b-II).

\section{Plant height (PH):}

A major effect QTL, qPH12-1, was identified between flanking markers, RM28275 (33.70 cM) and RM511 (41.66 cM) with LOD score of 2.72 and with PVE\% of $43.88 \%$. The additive effect value, -6.32 , of this QTL demonstrated the inheritance of the favorable allele from KMR-3R. The cumulative phenotypic effect (RSq value) of this QTL on trait accounted up to $41.08 \%$. (Additional file 1; Table 1, Figure 2 ). 
Table 1

Putative major effect QTLs for agronomic traits in 125 doubled haploid (DH) population derived from the cross of IR58025A $\times$ KMR-3R (KRH-2)

\begin{tabular}{|c|c|c|c|c|c|c|c|c|c|c|c|}
\hline $\begin{array}{l}\text { Trait } \\
\text { Name }^{a}\end{array}$ & $\mathrm{QTL}^{\mathrm{b}}$ & Flanking $\mathrm{m}$ & rkers & Chr & $\begin{array}{l}\text { Start } \\
\text { position } \\
\text { (cM) }\end{array}$ & $\begin{array}{l}\text { End } \\
\text { position } \\
\text { (cM) }\end{array}$ & $\begin{array}{l}\text { QTL Size } \\
\text { (cM) }\end{array}$ & $\begin{array}{l}\text { LOD } \\
\text { peak }^{\text {c }}\end{array}$ & $\operatorname{PVE}(\%)^{d}$ & Add $^{e}$ & $\mathrm{RSq}^{\mathrm{f}}$ \\
\hline DFF & $\underset{1}{q D F F 12-}$ & RM27966 & RM235 & 12 & 46.68 & 51.69 & 5.01 & 3.64 & 48.60 & 9.77 & 51.01 \\
\hline \multirow[t]{2}{*}{ YLD } & $q Y L D 3^{S_{-1}}$ & RM448 & RM15679 & 3 & 93.41 & 113.12 & 19.71 & 16.51 & 56.75 & -7.81 & 97.26 \\
\hline & $q Y L D 6^{\#}-1$ & RM7023 & RM586 & 6 & 53.95 & 69.10 & 15.15 & 14.36 & 35.29 & 7.43 & - \\
\hline \multirow[t]{2}{*}{ TGW } & qTGW6-1 & RM19410 & RM19429 & 6 & 25.11 & 27.50 & 2.39 & 4.19 & 46.78 & -2.11 & 72.94 \\
\hline & qTGW7-1 & RM20948 & RM21649 & 7 & 167.52 & 172.53 & 5.01 & 3.06 & 29.95 & -1.24 & - \\
\hline PW & $q P W 9-1$ & RM23959 & RM23958 & 9 & 95.82 & 110.97 & 15.15 & 3.44 & 33.19 & -6.46 & 46.42 \\
\hline $\mathrm{PH}$ & $q P H 12-1$ & RM28275 & RM511 & 12 & 33.70 & 41.66 & 7.96 & 2.72 & 43.88 & -6.32 & 41.08 \\
\hline FLL & $q F L L \sigma^{\#-1}$ & RM7023 & RM586 & 6 & 53.95 & 69.10 & 15.15 & 3.09 & 30.67 & 4.06 & 42.89 \\
\hline FLW & $q F L W 4-1$ & RM252 & RM3524 & 4 & 7.96 & 10.46 & 2.5 & 3.29 & 51.18 & 0.17 & 48.22 \\
\hline \multirow[t]{2}{*}{ PL } & $q P L 3^{S-1}$ & RM448 & RM15679 & 3 & 93.41 & 113.12 & 19.71 & 16.51 & 56.75 & -7.80 & 97.26 \\
\hline & $q P L 6^{\#}-1$ & RM7023 & RM586 & 6 & 53.95 & 69.10 & 15.15 & 14.36 & 35.29 & 7.43 & - \\
\hline BM & $q B M 4-1$ & RM1388 & RM17162 & 4 & 56.87 & 68.68 & 11.81 & 2.70 & 37.79 & 8.31 & 47.32 \\
\hline
\end{tabular}

aDFF-Days to fifty percent flowering, YLD-Total grain yield per plant, TGW-1,000 grain weight, PW-Panicle weight, PH-Plant height, FLL-Flag leaf length, FLW-Flag leaf width, PL-Panicle length, BM-biomass

${ }^{\mathrm{b}}$ Designation of the QTLs is in accordance with the rules recommended by McCouch (2008)

'Logarithm of the odds ratio (LOD) score of $\geq 2.5$ was set as threshold for this data

${ }^{\mathrm{d}}$ Total phenotypic variance (PVE\%) in percentage explained by the QTL

${ }^{\text {e} A d d i t i v e ~ e f f e c t-N e g a t i v e ~ a d d i t i v e ~ e f f e c t ~ v a l u e ~ i n d i c a t e s ~ t h e ~ d i r e c t i o n ~ o f ~ f a v o r a b l e ~ a l l e l e ~ f r o m ~ d o n o r ~ p a r e n t, ~ K M R-3 R, ~ t h a t ~ i n c r e a s e d ~ t h e ~ t r a i t ~}$ value

${ }^{f}$ Phenotypic variation explained by the final regression model

\$QTL hotspot on chromosome 3 for YLD and PL, \# QTL hotspot on chromosome 6 for YLD, FLL and PL

\section{Flag leaf length (FLL):}

A major effect QTL for this trait, qFLL6-1, was observed to be flanked by RM7023 (53.95 cM) and RM586 (69.10 cM) with RM7023 being the closely associated marker. The size of the QTL was $15.15 \mathrm{cM}$, with a LOD score of 3.09 and explaining $30.67 \%$ of PVE. The inheritance of this QTL was from IR58025A with an additive value of 4.06. As per the RSq value, this QTL accounted up to $42.89 \%$ of cumulative phenotypic variance (Additional file 1; Table 1, Figure 2).

\section{Flag leaf width (FLW):}

qFLW4-1, a novel major effect QTL with a LOD score of 3.29 with PVE\% of $48.22 \%$ was identified in between the flanking markers, RM252 (7.96 cM) and RM3524 (10.46 cM) and the marker RM3524 to be closest to the QTL. The $2.5 \mathrm{cM}$ size QTL was observed to be inherited from IR58025A with an additive effect value of 0.17 and accounting up to $48.22 \%$ of the cumulative phenotypic variance (RSq) (Additional file 1 ; Table 1, Figure 2).

\section{Panicle length (PL):}


Two major effect QTLs, qPL3-1 and qPL6-1, were identified with a LOD score of 16.51 and 14.36 , respectively and the PVE\% for was observed to be $56.75 \%$ and $35.29 \%$, respectively. The 19.71 cM sized QTL, qPL3-1, was flanked by RM448 (93.41 cM) and RM15679 (113.12 cM), with RM15679 being the closely associated marker. A negative additive effect value of -7.80 indicated that the inheritance of the favorable allele of this QTL was from KMR-3R. qPL6-1, (15.15 cM sized) was observed to be flanked by RM7023 (53.95 cM) and RM586 (69.10 cM) and RM7023 being the closest marker. The additive effect value of 7.43 indicated that the inheritance of this QTL was from IR580525A. The cumulative phenotypic variance (RSq) of both these QTLs was observed to $97.26 \%$ indicating that they are major QTLs (Additional file 1; Table 1, Figure 2, Supplementary Figure 1a-(I)-1b (I).

\section{Biomass (BM):}

A novel biomass QTL, qBM4-1, (11.81 cM sized QTL) between RM1388 (56.87 cM) and RM17162 (68.68 cM) was identified with a LOD score of 2.70 and PVE\% of $37.79 \%$, with closest associated marker being, RM1388. The additive effect value of this QTL, 8.31, demonstrated its inheritance from IR58025A with an RSq value of 47.32 (Additional file 1; Table 1, Figure 2, Supplementary Figure 3a-II). The details of colocalization of major effect QTLs with earlier studies is presented in Additional file 1; Table 2. 
Table 2

Co-validation of putative major effect QTLs for various agronomic traits in 125 doubled haploid (DH) population derived from the cross of IR58025A × KMR-3R (KRH-2) with previous studies

\begin{tabular}{|c|c|c|c|c|c|c|c|c|c|c|}
\hline \multirow[t]{3}{*}{$\begin{array}{l}\text { Trait } \\
\text { Name }^{\mathrm{a}}\end{array}$} & \multirow{3}{*}{$\begin{array}{l}\mathrm{QTL}^{\mathrm{b}} \\
\\
q D F F 12- \\
1\end{array}$} & \multicolumn{2}{|c|}{ Flanking markers } & \multirow{2}{*}{$\begin{array}{l}\text { Chr. } \\
12\end{array}$} & \multirow{2}{*}{$\begin{array}{l}\text { QTL } \\
\text { position(s) } \\
\text { in this } \\
\text { study } \\
\text { (cM/Mb) } \\
\\
\begin{array}{l}12.16^{-} \\
26.17 \mathrm{Mb}\end{array}\end{array}$} & \multirow{2}{*}{$\begin{array}{l}\text { Published } \\
\text { QTLs } \\
\text { names in } \\
\text { previous } \\
\text { study } \\
\\
\text { qt/12.1 }\end{array}$} & \multicolumn{2}{|c|}{$\begin{array}{l}\text { Flanking marker(s) in } \\
\text { previous study }\end{array}$} & \multirow{2}{*}{$\begin{array}{l}\text { Published } \\
\text { QTL } \\
\text { position(s) } \\
\text { in } \\
\text { previous } \\
\text { study } \\
\text { (cM/Mb) } \\
\text { 7.46-17.44 } \\
\text { Mb }\end{array}$} & \multirow{2}{*}{$\begin{array}{l}\text { Previous } \\
\text { study } \\
\text { Bernier et al. } \\
(2007)^{72}\end{array}$} \\
\hline & & RM27966 & RM235 & & & & RM3103 & RM511 & & \\
\hline & & & & 12 & $\begin{array}{l}12.16- \\
26.17 \mathrm{Mb}\end{array}$ & $\mathrm{qDF}_{12.1}$ & id12006721 & 12884952 & $20 \mathrm{Mb}$ & $\begin{array}{l}\text { Descalsota- } \\
\text { Empleo et } \\
\text { al. }(2019)^{73}\end{array}$ \\
\hline \multirow[t]{2}{*}{ YLD } & $\begin{array}{l}q Y L D 3- \\
1\end{array}$ & RM448 & RM15679 & 3 & $\begin{array}{l}34.49- \\
26.87 \mathrm{Mb}\end{array}$ & GY QTL & RM416 & & $31.24 \mathrm{Mb}$ & $\begin{array}{l}\text { Agrama et } \\
\text { al. }(2007)^{74}\end{array}$ \\
\hline & $\begin{array}{l}q Y L D 6- \\
1\end{array}$ & RM7023 & RM586 & 6 & $\begin{array}{l}6.98-1.47 \\
\mathrm{Mb}\end{array}$ & qGY6-1 & RM217 & & $4.05 \mathrm{Mb}$ & $\begin{array}{l}\text { Dejun et al. } \\
(2002)^{75}\end{array}$ \\
\hline \multirow[t]{2}{*}{ TGW } & $\begin{array}{l}\text { qTGW6- } \\
1\end{array}$ & RM19410 & RM19429 & 6 & $\begin{array}{l}2.91 \mathrm{Mb}- \\
3.41 \mathrm{Mb}\end{array}$ & qTGW6-1 & RM6273 & RM204 & $\begin{array}{l}0.13-3.16 \\
\mathrm{Mb}\end{array}$ & $\begin{array}{l}\text { Marathi et } \\
\text { al. }(2012)^{50}\end{array}$ \\
\hline & $\underset{1}{q T G W 7-}$ & RM20948 & RM21649 & 7 & $\begin{array}{l}2.5-18.8 \\
\mathrm{Mb}\end{array}$ & gw7.1 & RM125 & & $5.46 \mathrm{Mb}$ & $\begin{array}{l}\text { Septiningsih } \\
\text { et al. } \\
(2003)^{76}\end{array}$ \\
\hline PW & $q P W 9-1$ & RM23959 & RM23958 & 9 & $\begin{array}{l}95.82- \\
110.97 \mathrm{cM}\end{array}$ & - & - & - & - & Novel QTL \\
\hline $\mathrm{PH}$ & $\begin{array}{l}q P H 12- \\
1\end{array}$ & RM28275 & RM511 & 12 & $\begin{array}{l}14.1-17.6 \\
\mathrm{Mb}\end{array}$ & - & - & - & - & Novel QTL \\
\hline FLL & $q F L L 6-1$ & RM7023 & RM586 & 6 & 6.9-1.4 Mb & QFII6 & RM588 & RM589 & $\begin{array}{l}1.61-1.38 \\
\mathrm{Mb}\end{array}$ & $\begin{array}{l}\text { Bing et al. } \\
(2006)^{77}\end{array}$ \\
\hline FLW & $\underset{1}{q F L W 4-}$ & RM252 & RM3524 & 4 & $\begin{array}{l}24-22.8 \\
\mathrm{Mb}\end{array}$ & - & - & - & - & Novel QTL \\
\hline \multirow[t]{2}{*}{ PL } & qPL3-1 & RM448 & RM15679 & 3 & $\begin{array}{l}93.41- \\
113.12 \mathrm{cM}\end{array}$ & - & - & & - & Novel QTL \\
\hline & qPL6-1 & RM7023 & RM586 & 6 & 6.9-1.4 Mb & qPL6.1 & YJInDel-207 & $\begin{array}{l}\text { YJInDel- } \\
208\end{array}$ & $\begin{array}{l}5.2-7.9 \\
\mathrm{Mb}\end{array}$ & $\begin{array}{l}\text { Kinoshita et } \\
\text { al. }(2017)^{78}\end{array}$ \\
\hline BM & $q B M 4-1$ & RM1388 & RM17162 & 4 & $\begin{array}{l}56.87- \\
68.68 \mathrm{cM}\end{array}$ & - & - & - & - & Novel QTL \\
\hline
\end{tabular}

A total of 12 minor effect QTLs were identified for the following traits: fertile grains per panicle (FGP), panicle weight (PW) and panicle length (PL). For the trait FGP, a total of nine QTLs namely, qFGP4-1, qFGP5-1, qFGP6-1, qFGP6-2, qFGP8-1, qFGP8-2, qFGP8-3, qFGP9-1, qFGP12-1, whose LOD scores were in the range of $2.66-4.25$ with $P V E \%$ in the range of $9.06 \%-9.78 \%$ were identified. The cumulative phenotypic effect (RSq) of all these QTLs on the trait accounted up to 45.89\%. Two minor effect QTLs for trait panicle weight (PW), $q P W 3-1$ and $q P W 8-1$, were identified with LOD scores, 2.60 and 3.44, respectively with PVE\% of $17.90 \%$ ( $q P W 3-1$ ) and $18.56 \%$ ( $q P W 8-1)$. The RSq value of these two QTLs accounted up to 46.42. qPL8-1, a minor effect QTL for panicle length trait was identified with a LOD score of 7.56 and PVE\% of $7.08 \%$. The negative additive effect value of -3.74 demonstrated the inheritance of favorable allele from KMR-3R. The cumulative phenotypic variance (RSq) value of this QTL with two major effect QTLs accounted up to 97.26\%. The details of these minor effect QTLs is shown in Supplementary Table 7. The details of co-localization of minor effect QTLs from earlier studies are presented in Supplementary Table 8. 
Epistatic interactions of QTLs with LOD peak > 2.5 and PVE\% > 20\% can be considered as significant and major effect in nature. Significant epistatic interactions were observed between the QTLs located on chromosomes 2, 3, 4, 6 and 11 and controlling three traits namely, days to fifty percent flowering (DFF), panicle weight (PW) and flag leaf length (FLL) (Additional file 1; Table 3, Supplementary Figure 2). For the trait DFF, epistatic interaction was observed between the QTLs located on chromosomes 11 and 12 with LOD score of 6.63 and PVE\% accounting up to $79.58 \%$. The RSq value of this significant epistatic interaction was observed up to $51.02 \%$. Similarly, for the trait panicle weight (PW), complex epistatic interactions were observed as shown in Supplementary Figure 2. Among these, three significant QTL epistatic interactions were observed between the QTLs located on chr $2-6$, chr $6-7$ and chr $3-9$, with total phenotypic variance (RSq) accounting for $46.4 \%$. Epistatic interaction was also observed between QTLs located on chr 4 and 12 for the trait flag leaf length (FLL) with a LOD score of 5.25 and phenotypic variance (PVE\%) value of $78.26 \%$. As shown in Supplementary Figure 3, among the DHL population, two high yielding DHLs namely, DHL-1 (RP6301-189-17-2) and DHL-2 (RP6301-188-15-47) were observed to be best performing with $32.13 \mathrm{~g}$ and $31.28 \mathrm{~g}$ of total grain yield per plant (YLD), respectively. DHL-1 was observed to have longer panicles, more number of productive tillers and medium bold (MB) grain type whereas DHL-2 had longer-dense panicles with highly desirable medium slender (MS) grain type.

Table 3

Major effect epistatic interactions between QTLs in 125 doubled haploid (DH) population derived from the cross of IR58025A $\times$ KMR-3R (KRH-

\begin{tabular}{|c|c|c|c|c|c|c|c|c|c|c|c|c|}
\hline $\begin{array}{l}\text { Trait } \\
\text { Name }^{a}\end{array}$ & $\begin{array}{l}\text { Chr } \\
1\end{array}$ & $\begin{array}{l}\text { QTL } \\
\text { name }^{b}\end{array}$ & $\begin{array}{l}\text { Left } \\
\text { Marker } 1\end{array}$ & $\begin{array}{l}\text { Right } \\
\text { Marker } 1\end{array}$ & $\begin{array}{l}\text { Chr } \\
2\end{array}$ & $\begin{array}{l}\text { QTL } \\
\text { name }^{\text {b }}\end{array}$ & $\begin{array}{l}\text { Left } \\
\text { Marker } 2\end{array}$ & $\begin{array}{l}\text { Right } \\
\text { Marker } 2\end{array}$ & $\begin{array}{l}\text { LOD } \\
\text { peak }^{\mathrm{C}}\end{array}$ & $\operatorname{PVE}(\%)^{d}$ & $\begin{array}{l}\text { Add } \\
\text { by } \\
\text { Add }^{\mathrm{e}}\end{array}$ & $\mathrm{RSq}^{\mathrm{f}}$ \\
\hline DFF & 11 & $\begin{array}{l}q D F F 11- \\
1\end{array}$ & $\begin{array}{l}\text { Chr 11- } \\
28.1\end{array}$ & RM286 & 12 & $\begin{array}{l}q D F F 12- \\
1\end{array}$ & RM511 & RM27966 & 6.63 & 79.58 & -7.10 & 51.02 \\
\hline PW & 2 & $q P W 2-1$ & RM12434 & RM13709 & 6 & $q P W 6-1$ & RM19429 & Chr 6-21.4 & 5.11 & 24.49 & 5.66 & 46.43 \\
\hline PW & 6 & qPW6-2 & RM7023 & RM586 & 7 & $q P W 7-1$ & RM21941 & RM20775 & 6.55 & 20.29 & -1.15 & 0.00 \\
\hline PW & 3 & qPW3-1 & RM422 & RM16238 & 9 & $q P W 9-1$ & RM23959 & RM23958 & 5.90 & 49.14 & 4.05 & 0.00 \\
\hline FLL & 4 & $q F L L 4-1$ & RM1388 & RM17162 & 12 & $\underset{1}{q F L L 12-}$ & RM519 & RM27920 & 5.25 & 78.26 & 5.52 & 78.26 \\
\hline
\end{tabular}

a DFF-Days to Fifty percent Flowering; PW-Panicle weight; FLL-Flag leaf length

${ }^{\mathrm{b}}$ Designation of the QTLs is in accordance with the rules recommended by McCouch (2008)

'Logarithm of the odds ratio (LOD) score of $\geq 2.5$ was set as threshold for this data

dTotal phenotypic variance in percentage explained by the QTL

eAdditive by additive effect

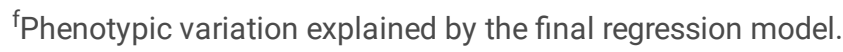

\section{QTL × environment}

QTL (genotype) x environment interaction of both major and minor effect QTLs in three consecutive seasons namely wet season (2014-2015), dry season (2015) and wet season (2015-2016) is presented in Supplementary Table 9. Some of the crucial parameters namely the LOD score for additive by environment effects (LOD(AbyE)), Phenotypic variation explained by additive by environment effect at the current scanning position (PVE(AbyE)), Additive by environment effect which determine the genotype $\times$ environment interactions (AbyE) were observed to zero in both major and minor effect QTLs. Cumulative phenotypic variance (RSq values) were also observed with least significant variation in all the identified QTLs.

\section{In-silico analysis of the major effect QTLs}

Supplementary Table 10 enlists the details of the putative candidate genes located in the vicinity of the physical positions of novel major effect QTLs identified through in-silico analysis. Pertaining to the physical positions of the plant height QTL, qPH12-1, out of 136 annotated genes in QTARO's database, three putative candidate genes with possible biological function related to trait phenotype were identified in the region around $17.57 \mathrm{Mb}$. Out of 1,344 annotated genes within the physical positions of panicle length, $q P L 3-1$, only one putative candidate gene located at $30.48 \mathrm{Mb}$, with possible biological function related to trait phenotype was identified. Similarly, out of 137 annotated genes identified within the physical positions of biomass QTL, $q B M 4-1$, only one putative candidate gene located at $25.10 \mathrm{Mb}$ position in the genome was

Page $9 / 20$ 
observed to have a close biological function with respect to the trait, biomass. Further, no candidate gene, having a possible biological role in regulating the QTLs viz., qPW9-1 and qFLW4-1 could be identified in the physical positions of these QTLs.

Molecular assessment of the DHL population with functional markers specific for $R f 3$ and $R f 4$ revealed the existence of four groups of DHLs (Supplementary Table 1). The first group comprised of 104 individuals was observed to have both fertility restoration loci. The second group consisting of nine individuals had $R f 3$ locus only. The third group consisted of 11 individuals of $R f 4$ locus only and one DHL was negative for both the loci.

\section{Discussion}

Large scale cultivation of rice hybrids is one of the feasible options for enhancing the rice production, amidst plateauing yield levels, diminishing natural resources and increasing population. It is estimated that through the adoption of this technology, the rice productivity could be increased by $35 \%-40 \%$ [55]. In India, out of a total 44.15 million hectares under rice cultivation, hybrid rice occupies less than three million hectares [4]. Limited adoption of hybrid rice in India could be primarily attributed to the lower yield advantage of these hybrids over the conventional varieties, coupled with lack of good grain quality features in rice hybrids $[5,56]$. Therefore, in order to popularize the hybrids, there is an imminent requirement that the released novel hybrids have at least $20-30 \%$ yield advantage in comparison with the existing varieties along with the appropriate supporting policies for their better adoption $[5,57,58]$. As opined by [7], development of such highly heterotic hybrids could be possible through the identification of genomic regions associated with yield heterosis and their targeted transfer to elite parental lines through molecular breeding.

Immortal populations viz., doubled haploid lines (DHLs), near isogenic lines (NILs), recombinant inbred lines (RILs) etc. can be employed for the identification of QTLs. Among the various populations, the development of complete homozygous population and fixation of the desired genetic background is possible in the shortest time only with DHLs [13]. Though utility of DHLs for varietal improvement and QTL mapping of various traits is well known [59], their application in QTL mapping for heterosis and yield associated QTLs is limited. In the present study, using a doubled haploid (DH) population developed from the cross IR58025A/KMR3R and by employing SSR and EST-derived SSR markers, new regions in the rice genome, associated yield and its other trait components have been mapped.

\section{Statistical analysis of the population}

F statistic, an important parameter in the combined ANOVA analysis demonstrated that each trait contributed for the significant portion of the variability in the DHL population. The population was analyzed with the help of various important statistical parameters namely, the standard deviation, W-value, coefficient of variation (\%), P-value, skewness, kurtosis with respect to the 12 traits under study. A very high standard deviation was observed for the traits, GP and FGP which indicated towards a high degree of variation among the DHLs. As the W-value of all the traits was observed to be nearly 1 , this indicated the uniformity among the replications. A moderate percentage of coefficient of variation for all the traits, except DFF indicated existence of sufficient agro-morphological variability in the population. Statistical P value was observed to be typically $\leq 0.05$ for most of the traits, except FLW indicating significance and appropriateness of the data used for statistical analysis. Univariate distribution of the data was indicated through the skewness values which were observed to be in the range -1.96 and $+1.96[60$, 61]. Normal distribution of the data was indicated with kurtosis values less than 3 . Estimation of genetic variability in the DHL population demonstrated a higher phenotypic variance (Vp)-phenotypic coefficient of variance (PCV) than genotypic variance (Vg)-genotypic coefficient of variance (GCV) indicating a higher environmental influence on the traits. Moreover, the magnitude of the difference between Vp-PCV and VgGCV was observed to be high, which again indicated towards the environmental predominance on the traits as compared to the genetic factors. These observations are in accordance with [62]. Therefore, for crop improvement, a study of genetic and non-genetic factors namely, Vp-PCV and Vg-GCV, is a reliable estimate for enhancing the breeding value along with selective breeding [63].

Heritability estimates are crucial for determining the contribution of both inheritable and non-inheritable factors for crop improvement. As suggested by [63], heritability is one of the reliable tools for estimating the breeding value on the basis of phenotypic evaluation. High broad sense heritability $\left(H^{2}\right)$ was observed for traits namely, GP, FGP, PW, PL and BM, demonstrating possibility of selection mediated improvement of these traits as reported earlier by $[64,65]$. Genetic advance (GA) under one cycle of selection at specific intensity is an indicator of increment of genotypic value of a trait. As opined by $[62,66]$, the genetic gain under selection for quantitatively inherited traits could be understood when high heritability $\left(H^{2}\right)$ is combined with high genetic advance per mean values (GAM). In our study, high $H^{2}$ values along with high GAM values for traits GP, FGP and BM along with moderate $H^{2}$ values along with moderate GAM values for traits $\mathrm{PW}, \mathrm{PH}, \mathrm{PL}$ and YLD demonstrated the role of additive gene action and development of breeding lines with higher GP, FGP and BM during early selections would be beneficial [62]. With respect to the traits, TGW, FLW and PT moderate to low $H^{2}$ values along with low GAM value demonstrated non-additive gene action in play and development of varieties with higher TGW, FLW and PT could be possible only through recurrent selection [62, 67]. 
Correlation is an indirect tool of selection that gives a deeper understanding of the degree and direction of selection. As pointed out by Nor et al. (2014)[68], correlation is important for determining the traits to be chosen for selection and improvement. In our study, we observed a significant and positive correlation among the YLD and other yield related traits namely, TGW, FLL, FLW, PW, PT and GP at both $1 \%$ and 5\% level of significance. Earlier studies viz., $[68,69,70]$ confirmed our results as well. A very strong-positive correlation was noted among TGW and PW, PT, PL and BM. Based on the results obtained from this study, the agro-morphological traits, YLD along with TGW, PW, FLL, FLW, PT and $\mathrm{GP}$ can be considered for varietal/parental line improvement.

\section{ICIM based QTL mapping}

Thomson et al. (2003)[71], in order to address the issue of lack of reliability and consistency of identified QTLs (particularly when the same population was employed for QTL mapping across various environments) suggested the inclusion of an empirical threshold value for consistent and robust QTL identification along with the consideration of data generated through multiple replicates. Keeping this in view, the agro-morphological data for yield and its allied traits was collected across different seasons with atleast ten plants of each DHL entry.

Consistency in the QTLs was confirmed by comparing the genomic regions of the QTLs identified. Out of the 24 QTLs identified, 16 of them were reported by previous research groups involving various types of populations along with employment of different types of markers for QTL mapping. A total of eight novel QTLs including five major effect QTLs associated with panicle weight ( $q P W 9-1)$, panicle length ( $q P L 3-1)$, flag leaf width ( $q F L W 4-1)$, plant height ( $q P H 12-1)$, biomass ( $q B M 4-1)$ and three minor effect QTLs for panicle weight ( $q P W 3-1$ and $q P W 8-1)$ and fertile grains per panicle ( $q F G P 5-1)$ were identified.

At least one QTL for each trait was identified in this study, except for the traits, GP and PT. It was interesting to observe that majority (58.33\%) of the identified QTLs were from IR58025A parent. A total of 14 QTLs for various traits namely DFF, YLD, FGP, PW, FLL, FLW and BM were contributed by IR58025A. Likewise, a total of 10 QTLs for traits viz., YLD, TGW, PW, PH, PL and FGP were contributed by KMR-3R. As pointed out by Marathi et al. (2012), these observations indicated towards the phenotypic and allelic variation existing among the parental lines and the possibility of combining these QTLs in a single parent for significant enhancement of yield and heterosis levels.

\section{Major effect QTLs co-localized with those from earlier reports}

Seven out of the 12 major effect QTLs identified in this study were observed to overlap with the genomic region of the QTLs mapped in earlier studies. For example, qDFF12-1 (12.16-26.17 Mb) co-localized with the QTL identified by [72, 73]. Another major QTL, qYLD3-1 (34.49 Mb26.87 Mb) co-localized with the yield (GY) QTL identified by [74]. Similarly, qYLD6-1 (6.98 Mb-1.47 Mb) was noted to co-localize with the QTL, qGY6-1, as reported by [75]. The QTL for 1,000 grain weight, qTGW6-1 (2.91 Mb-3.41 Mb), overlapped with the QTL region controlling grain weight reported by [50]. The second major effect QTL for TGW trait, qTGW7-1 (2.52 Mb-18.81 Mb), identified was earlier resolved to a 5.46 Mb region on chr 7 by [76]. A major QTL for flag leaf length, qFLL6-1(6.98 Mb-1.47 Mb), identified, co-localized with another QTL identified earlier for the same trait, qF116 [77]. The panicle length QTL, qPL6-1 (6.98 Mb-1.47 Mb), was in the same genomic region where another QTL for the same trait, qPL6.1 was located [78].

\section{Novel major and minor QTLs identified}

Five major effect QTLs for the traits, panicle weight ( $q P$ W9-1), panicle length ( $q P L 3-1)$, flag leaf width ( $q F L W 4-1)$, plant height ( $q P H 12-1)$ and biomass ( $q B M 4-1)$ were identified in genomic regions where no other QTLs for the same trait has been reported, hence can be considered novel. It is indeed pertinent to note that all the novel QTLs are associated with traits of agronomical importance and hence may have significant utility in breeding programs. We are developing QTL-near isogenic lines (QTL-NILs), in order to fine-map novel major QTLs and identify the causative genes underlying these QTLs. In addition, to the major QTLs, a total of three minor effect QTLs were identified to be novel in this study. Also, since two high yielding DHLs namely DHL-1 and DHL-2 were observed to have longer panicles with more number of productive tillers and dense panicles, these lines may have novel major effect QTLs, viz., qPL3-1, qPW9-1.

\section{QTL hotspots}

A QTL hotspot was identified in the genomic region on chromosome 3, flanked in between RM448 (34.49 Mb) and RM15679 (26.87 Mb), wherein two major effect QTLs viz., $q Y L D 3-1$ and $q P L 3-1$ were located. A part of this QTL hotspot, i.e. physical position, 31.24 Mb, was earlier reported by [74] to possess a major QTL governing yield (GY) trait. As the two traits, PL and GY are positively correlated to each other [56, 79], it is possible that a single candidate gene could be controlling both the traits. A second QTL hotspot region flanked within the SSR markers, RM7023 (6.98 Mb) and RM586 (1.47 Mb) on chr 6 was observed to govern three traits namely YLD (qYLD6-1), FLL (qFLL6-1) and PL (qPL6-1) which are interrelated to each other functionally. Concerning the minor effect QTLs, one QTL hotspot on chr 8 at physical position $24.18 \mathrm{Mb}$ was noted to be associated with that traits, panicle weight and panicle length, traits which may not be related to each other at functional level.

The population containing (125 individuals DH lines ) employed in this study could be considered as optimal, as some previous research groups viz., [73, 74, 75, 80, 81], mapped yield QTLs using a similar sized population and using various set of molecular markers. [74] used 92 
lines for association mapping of four yield and its related traits. [80] used $96 \mathrm{BC}_{2} \mathrm{~F}_{2}$ progenies for phenotypic evaluation and $93 \mathrm{BC} \mathrm{F}_{1}$ plants for linkage map construction for eleven yield and its related trait components. [75] used 90 individuals of $\mathrm{BC}_{4} \mathrm{~F}_{1}$ population was used for $\mathrm{QTL}$ mapping of six yield and its related traits. [73] employed 111 DHLs for QTL mapping of three yield and its related trait components. [81] used 81 DHLs for mapping five yield and its related traits.

\section{In-silico analysis of novel major effect QTLs}

Putative candidate gene (s) could be identified for three major effect QTLs viz., $q P H 12-1, q P L 3-1$ and $q B M 4-1$. For the plant height QTL, $q P H 12-$ 1, three candidate genes with RAP-DB locus IDs viz., Os12t0479400-01, Os12t0479400-02 and Os12t0479400-03, were identified at 17.57 Mb on chromosome 12. These three genes were observed to encode different transcriptional factors namely transcriptional factor B3 (IPR003340) and Aux/IAA-ARF-dimerization protein (IPR011525) that are associated with expression of AUX/IAA protein (IPR003311) (Rice Annotation Project Database (RAP-DB) (https://rapdb.dna.affrc.go.jp/). As plant growth hormones, Auxin/Indole Acetic Acid (IAA) are well characterized and are known to regulate the overall plant architecture including the plant height $[82,83]$, the putative candidate genes underlying the QTLs may be influencing plant height. Productive tillers and morphology of the panicle influence the total grain yield/plant (YLD) architecture in rice. The panicle morphology is further influenced by length and weight of the panicle. Biomass (BM) trait has also been well documented to influence the YLD in plant and therefore YLD's genetic architecture. In our study, using the DHL population consisting of 125 individuals and employing 126 hyper-variable SSRs and EST-derived SSRs, novel QTLs for traits panicle length ( $q P L 3-1)$ and biomass ( $q B M 4-1)$ were assessed for the identification of putative candidate genes. Two putative candidate genes, with RAP-DB locus ID viz., Os03t0742900-03 and Os03t0742900-01 associated with panicle length QTL, qPL3-1, were also observed to be Auxin (AUX)/IAA response proteins that could be controlling panicle length. A putative candidate gene identified with biomass QTL, qBM4-1, with RAP-DB locus ID: Os04t0491700-01 was observed to be a sugar/inositol transporter (IPR003663). As physiological transportation of sugar (s) from source to sink is crucial in plant biomass accumulation, this gene may have a role in augmenting the trait value of the biomass QTL identified in this study. Hence, as the molecular functions of the above mentioned putative candidate genes (s) are identifiable with the newly identified QTLs, it is conjectured that they might have a role in regulating the trait expression and therefore influencing the genetic architecture.

As the selection efficiency of the functional markers, specific for $R f 4$ and $R f 3$ was observed to be $83.4 \%$ and $52.5 \%$, respectively, it can be concluded that the presence of $R f 4+R f 3$ loci or $R f 4$ locus alone would impart the line with complete restoration potential. However, presence of only $R f 3$ locus would confer partial restoration capability, as reported in earlier studies [84, 85]. In our study, using these functional markers, 104 DHLs were observed to possess both the fertility restoration loci, 11 DHLs had only one major fertility restoration loci i.e., Rf4 and rest all were partial restorers. It would be interesting to envisage test-crosses between some of the selected high yielding and better performing DHLs with popular cytoplasmic male sterile (CMS) lines, to validate their complete fertility restoration potential.

\section{Conclusion}

Using doubled haploid lines (DHL) developed from the cross IR58025A/KMR3R, a total of 24 QTLs, comprising of 12 each of major and minor effect QTLs controlling agro-morphological traits associated with yield and allied parameters have been identified. Five novel major effect QTLs controlling the yield related traits viz., panicle length, panicle weight, plant height, flag leaf width, biomass with high LOD values and high Rsq values have been detected. Two major effect QTL hotspot regions on chromosome 3, governing total grain yield per plant (YLD)-panicle length (PL) and on chromosome 6 regulating YLD, PL and flag leaf length (FLL) have also been identified. The major QTLs and QTL hotspot regions identified in this study, could be transferred through marker assisted backcross breeding (MABB) into various genetic backgrounds.

\section{Methods}

\section{Experimental material}

The seeds of the popular, medium duration rice hybrid, KRH-2, developed from a cross between IR58025A and KMR-3R, with long-bold grain type, released for the irrigated ecology, with a high yield potential were obtained along with its parents from the Hybrid Rice Section, Indian Institute of Rice Research (IIRR), Hyderabad, India and were used as the experimental material. Varietal checks namely Akshayadhan (AKD) and Varadhan (VRD) were used as controls and their seeds were also obtained from the above mentioned section of IIRR, Hyderabad, India. Crosses were effected between cytoplasmic male sterile (CMS) line, IR58025A and the elite restorer, KMR-3R to produce the hybrid KRH-2 in the dry season 2014. As IR58025A does not set seeds due to sterile pollen, IR58025B was used for morpho-agronomic assessment of the DHL population. Hence, IR58025A was used as the female parent. IR58025B was used for the purpose of agro-morphological evaluation of the DHL population as IR58025A does not set seeds.

\section{Agro-morphological evaluation of developed DHLs}

Page 12/20 
Following the anther culture protocol standardized by [52], a total of 125 regenerated true, fertile and highly stable DHLs $\left(\mathrm{D}_{0}\right)$ were produced at the end of wet season 2015-2016. The DHL population was grown for three consecutive seasons (dry season 2016 , $D_{1}$ generation; wet season 2016-2017, $D_{2}$ generation; dry season $2017, D_{3}$ generation) for analyzing their genetic variability with respect to key agro-morphological traits and to assess the extent of correlation among yield and its contributing traits. For phenotyping of the DHL population, randomized complete block design (RCBD) with two replications was followed. Five middle plants from each DH line of each replication were selected and phenotyped. Hence, ten plants (i.e., from two replications) were considered for phenotypic evaluation. At appropriate growth stage of experimental material, 12 traits namely, days to fifty percent flowering (DFF), total number of grains per panicle (GP), test $(1,000)$ grain weight (TGW), number of productive tillers (PT), biomass (BM), fertile grains per panicle (FGP), panicle weight (PW), panicle length (PL), flag leaf length (FLL), flag leaf width (FLW), plant height (PH) and total grain yield per plant (YLD) were recorded from five healthy plants of middle row of each line (of each replication), as per the standard evaluation system recommended by [86] and the collected data was considered for further analysis. The procedure of recording these traits was followed as described in [87].

\section{Statistical analysis}

Mean of ten plants of each DH line across three seasons was considered for analysis of statistical parameters namely, frequency distribution for agro-morphological traits, standard deviation, coefficient of variation in percentage (CV\%), W value, probability value (P-value), skewnesskurtosis and were calculated using Statistical Tool for Agricultural Research (STAR) software version 2.0.1, IRRI. Using SAS version 9.3 (SAS Institute Inc., Cary, NC, USA), combined one way analysis of variance (ANOVA) of three seasons' morpho-agronomic data, genotypic-phenotypic correlation analysis among all the agro-morphological traits and genetic variability estimates for yield related traits was done.

\section{Extraction of genomic DNA and genotyping of the DHL population with hyper- variable SSRs}

The genomic DNA was isolated from fresh and healthy leaves of $125 \mathrm{DHLs}\left(\mathrm{D}_{3}\right.$ generation) and parents IR58025A-KMR-3R, following the protocol of CTAB MiniPrep method described by [88]. The protocol for setting up the polymerase chain reaction (PCR) including the PCR profile was followed as described in [87]. The products amplified after the PCR were resolved in 4\% agarose gel (Sigma, USA) using Protean II gel casting and electrophoresis apparatus (BioRad, USA). As the agarose gel was stained with $0.5 \mu \mathrm{g} / \mathrm{ml}$ ethidium bromide, visualization of the amplified bands was done using a gel documentation system (Alpha Innotech, USA). A total of 1,904 SSR markers were used for polymorphism survey among which 134 polymorphic markers between the parents were used for genotyping the DHL population. Using segregation distortion loci (SDL) mapping function of IciMapping software ver. 4.0.1 [89, 90] (https://www.isbreeding.net), out of these 134 SSR markers, eight markers, namely, RM13616 (chromosome 2), RM15370 (chromosome 3), RM6697 (chromosome 4), RM7449 (chromosome 5), RM18371 (chromosome 5), RM19402 (chromosome 6), RM21643 (chromosome 7), RM25502 (chromosome 10) were identified with segregation distortion. Therefore, these eight distorted markers, 5.97\% out of 134 polymorphic markers were omitted in the mapping of QTLs mapping exercise. Thus, 126 SSRs with no segregation distortion were only employed for QTL linkage map construction. The details of genomic SSRs are available at GRAMENE (www.gramene.org) while the EST-derived SSR markers were selected from [91]. Manual allele scoring was done as per the expected allele sizes enlisted in GRAMENE (www.gramene.org) and in [91]. Those bands which had unambiguous amplification were scored with $50 \mathrm{bp}$ ladder. The alleles of the amplified DHLs with the SSR markers were scored as 1 if an expected size of the amplicon is observed and as 0 for the absence of the expected amplicon size. Hence, a binary data-matrix based on the above mentioned scheme of scoring was used for QTL mapping.

\section{Percent of missing data-points among the SSR and EST-derived markers}

In order to determine the percentage of the missing data-points using the SSR and EST-derived SSRs, the number of data-points expected to amplify with 126 hyper-variable SSRs in the DHL population was computed on the basis of the number of DH individuals used for QTL mapping. Then, the number of data-points that did not amplify was recorded. The ratio of unamplified data-points to the total number of amplified data-points was multiplied with 100 to obtain percentage.

\section{Construction of QTL map}

Inclusive composite interval mapping method (ICIM) was employed for the construction linkage map using binary data matrix of hyper-variable SSRs with IciM ver. 4.0.1 [89, 90] (https://www.isbreeding.net). The Kosambi map function was used for mapping the QTLs using the above software and determination of significant QTLs $(P=0.05)$ was done by setting the permutation number to 1,000 with a minimum LOD threshold value of 2.5 and the range of LOD value being 2.5-3.3. Upon the detection of significant LOD peak with a minimum value of 2.5, the values of various other factors which influence the QTL mapping viz., the log-likelihood ratio (LOD), phenotypic variation explained (PVE) in percentage and additive effect were determined. The statistical identification of the QTLs based on the LOD score was done as per the procedure described in [92]. As mentioned in [54], the QTLs were termed as major effect if their phenotypic variation percentage (PVE\%) was more than 20\%. QTL nomenclature was followed as described by [93]. Manual identification of the QTLs was done as per the procedure described in Marathi et al. (2012) but with slight modifications. The QTL hotspots were identified by searching the QTL linked to the genomic

Page $13 / 20$ 
regions with in a window size of $20 \mathrm{cM}$ wherein two or more QTLs were observed to be co-located within the each window region. The construction of linkage QTL map, determination of epistatic (digenic) interactions between pairs of markers was done using QTL IciMapping software ver. 4.0.1 $[89,90]$. For determining the quantitative epistatic interactions (QEls) among the identified QTLs, an LOD threshold value of 3.0 was set as a minimum. In order to determine the co-factors of QTL mapping namely, the LOD values, PVE\% and additive effect, the stepwise regression analysis was used. For estimating the QTL whether is a major or minor effect QTL, maximum-likelihood method was used. Using IciM, the interaction between QTL (genotype) and environment was determined using the MET functionality. For determining whether the QTLs detected through this study were earlier reported by other research groups or novel, the physical positions of these QTLs were compared to those identified in earlier reports from the GRAMENE QTL database (http://archive.gramene.org/qtl/) and QTARO database (http://qtaro.abr.affrc.go.jp/qtab/table\#as_table:21:undefined:undefined). As described in [50], the QTLs identified through this study were reported to be novel if the physical positions of the SSR marker intervals did not match with those of the earlier studies. For identifying the functions of the putative candidate gene (s) within the physical positions of the novel major effect QTLs, the Rice Annotation Project Database (RAP-DB) (https://rapdb.dna.affrc.go.jp/) was used.

\section{Molecular assessment of fertility restoration loci in DHL population}

For identifying complete-partial restorers within the 125 DHLs without resorting to tedious test crosses, genomic DNA was isolated from freshyoung leaves of DHL plants along with the hybrid KRH-2 and its parents using the method described by [88]. The isolated genomic DNA was amplified employing the functional markers viz., RMS-SF21-5 and RMS-PPR9-1, specific for the minor-major fertility restoration loci, respectively [84].

\section{Abbreviations}

QTL-Quantitative Trait Loci; DHL-Doubled Haploid Lines; SSR-Simple Sequence Repeats; ICIM-Inclusive Composite Interval Mapping; KRH-2Karnataka Rice Hybrid-2; ZARS-Zonal Agricultural Research Station; KMR-3R-Karnataka Mandya Rice-3R; DFF-Days to Fifty percent Flowering (DFF); YLD-Total grain yield per plant; GP-Total number of grains per panicle; FGP-Fertile Grains per panicle; TGW-Test (thousand) Grain Weight; PW-Panicle Weight; PH-Plant Height; PL-panicle length; FLL-Flag Leaf Length; FLW-Flag Leaf Width; PT-Productive Tillers; BM-Biomass; CV\%Coefficient of Variation in percentage; STAR-Statistical Tool for Agricultural Research (STAR) software version 2.0.1; ANOVA-Analysis Of Variance; DNA-Deoxy Ribose Nucleic Acid; CTAB-Cetyl Trimethyl Ammonium Bromide; PCR-Polymerase Chain Reaction; Tris HClTrisaminomethane hydrochloric acid; $\mathrm{KCl}$-Potassium Chloride; $\mathrm{mM}$-milli Molar; $\mathrm{MgCl}_{2}$-Magnesium Chloride; dNTP-deoxyribonucleotide triphosphate; pmol-pico mole; pH-Potential of Hydrogen; ng-nano gram; U-unit; v/v-volume/volume; ${ }^{\circ} \mathrm{C}$-degree centigrade; EST-Expressed Sequence Tags; LOD-log-likelihood ratio; PVE-Phenotypic Variation Explained; cM-centi Morgan.

\section{Declarations}

\section{Ethical approval and consent to participate:}

Not applicable

\section{Consent for publication:}

Not applicable

\section{Availability of data and material:}

The data and information generated from the study are available in our laboratory as hard and soft copies and can be shared based on request.

\section{Competing interests:}

The authors declare that they have no competing interests

\section{Funding:}

DST INSPIRE Fellowship Division, New Delhi (Grant \# DST/INSPIRE Fellowship/2013/1146) and Department of Biotechnology (DBT), Govt. of India. 


\section{Authors' contributions:}

SRK carried out anther culture, developed the doubled haploid population, carried out the field studies, QTL mapping and drafted the manuscript; BSM provided the infrastructural facilities for doubled haploid production, for QTL mapping work and critically reviewed the manuscript; UK revised the manuscript; DB guided with the QTL mapping studies and revised the manuscript; HAS helped with the development of KRH-2 hybrid; RG, KMBVN, RRK, HG, AM, PE, DT, HSK, PK, AD, MS, CK, PS helped with field data collection for three consecutive seasons; KPR provided the initial $\mathrm{KRH}-2$ hybrid seed, SRM provided the lab and field infrastructural facilities for doubled haploid production, guided with the QTL mapping work and critically revised the manuscript. All authors read and approved the final manuscript.

\section{Acknowledgements:}

All the authors are grateful to the Director, ICAR-IIRR, Rajendranagar, Hyderabad, India, for providing the infrastructural facilities for carrying out this research work.

\section{References}

1. Gramaje LV, Caguiat JD, Oscar SJ, dela Cruz QD, Millas RA, et al. Heterosis and combining ability analysis in CMS hybrid rice. Euphytica. 2020;216(14):1-22.

2. Khush G. Strategies for increasing the yield potential of cereals: case of rice as an example. Plant Breed. 2003;132:433-6.

3. Virmani SS, Mao CX, Hardy B. Hybrid Rice for food security, poverty alleviation, and environmental protection. In: Proceedings of the 4th International Symposium on Hybrid Rice, Hanoi, Vietnam, 14-17 May 2002. 2002; International Rice Research Institute, Los Ban os, Philippines, p 407.

4. Senguttuvel P, Revathi P, Kemparaju KB, Sruthi K, Sadath Ali M, et al. Rice Hybrids Released in India. 2019;Compendium No. $103 / 2019$. ICAR-IIRR, Rajendranagar, Hyderabad-500 030. India. p. 127.

5. Spielman DJ, Kolady DE, Ward PS. The prospects for hybrid rice in India. Food Sec. 2013;5:651-65.

6. Virmani SS. Hybrid rice. Adv Agron. 1996;57:378-462.

7. Collard BCY, Cruz CMV, McNally KL, Virk PS, Mackill DJ. Rice molecular breeding laboratories in the genomics era: Current status and future considerations. J plant Geno. 2008. doi:10.1155/2008/524847.

8. Tinker NA, Mather DE, Rossnagel BG, Kasha KJ, Kleinhofs A, et al. Regions of the genome that affect agronomic performance in two-row barley. Crop Sci. 1996;36:1053-62.

9. Wang BN, Huang Z, Shu LH, Ren X, Li XH, et al. Mapping of two new brown plant hopper resistance genes from wild rice. Chin Sci Bull. 2001;46:1092-5.

10. Senadhira D, Zapata-Arias FJ, Gregorio GB, Alejar MS, De La Cruz HC, et al. Development of the first salt tolerant rice cultivar through indica/indica anther culture. Field Crops Res. 2002;76:103-10.

11. Suriyan C, Bootsaya S, Aussanee P, Chalermpol K. An efficient procedure for embryogenic callus induction and doubled haploid plant regeneration through anther culture of Thai aromatic rice (Oryza sativa L.subsp. indica). In Vitro Cell Dev Biol Plant. 2009;45(2):171-9.

12. Calayugan MIC, Formantes AK, Amparado A, Descalsota-Empleo GI, Nha CT, et al. Genetic Analysis of Agronomic Traits and Grain Iron and Zinc Concentrations in a Doubled Haploid Population of Rice (Oryza sativa L.). Sci Rep. 2020;10:2283. https://doi.org/10.1038/s41598020-59184.

13. Balachandran SM, Sarma NP, Siddiq E. Inheritance of anther culture response in rice. Curr Sci. 1999;77:962-4.

14. Kumar A, Singh VJ, Krishnan SG, Vinod KK, Bhowmick PK, et al. WA-CMS-based iso-cytoplasmic restorers derived from commercial rice hybrids reveal distinct population structure and genetic divergence towards restorer diversification. 3 Biotech. 2019;9:299,1-15.

15. Guo L, Gao Z, Qian Q. Application of resequencing to rice genomics, functional genomics and evolutionary analysis. Rice. $2014 ; 7: 4$.

16. Matsubara K, Yamamoto E, Kobayashi N, Ishii T, Tanaka J, et al Tsunematsu H, Yoshinaga S, Matsumura O, Yonemaru J, Mizobuchi R, Yamamoto T, Kato H, Yano M. (2016) Improvement of rice biomass yield through QTL-based selection. PLoS One. 2016;11: e0151830.

17. Segami S, Yamamoto T, Oki K, Noda T, Kanamori H, Sasaki H, et al. Detection of novel QTLs regulating grain size in extra-large grain rice (Oryza sativa L.) lines. Rice. 2016;9:34.

18. Xu Y, Li P, Yang Z, Xu C. Genetic mapping of quantitative trait loci in crops. Crop J. 2017;5:175-84.

19. Collard BCY, Jahufer MZZ, Brouwer JB, Pang ECK. An introduction to markers, quantitative trait loci (QTL) mapping and marker-assisted selection for crop improvement: The basic concepts. Euphytica. 2005;142:169-96. 
20. Raghavan C, Mauleon R, Lacorte V, Jubay M, Zaw H. Approaches in characterizing genetic structure and mapping in a rice multiparental population. Genes Genom Genet. 2017;G3:7:1721-30.

21. Singh A, Carandang J, Gonzaga ZJC, Collard BCY, Ismail AM, et al. Identification of QTLs for yield and agronomic traits in rice under stagnant flooding conditions. Rice. 2017;10:15.

22. Donde R, Kumar J, Gouda G, Gupta M, Mukherjee M, et al. Assessment of genetic diversity of drought tolerant and susceptible rice genotypes using microsatellite markers. Rice Sci. 2019;26:239-47.

23. Miura K, Ikeda M, Matsubara A, Song XJ, Ito M, et al. OsSPL14 promotes panicle branching and higher grain productivity in rice. Nat Genet. 2010;42:545-9.

24. Khush GS. Green revolution: preparing for the 21st century. Genome. 1999;42:10.

25. Gouda G, Gupta MK, Donde R, Kumar J, Vadde R, et al. Computational approach towards understanding structural and functional role of cytokinin oxidase/dehydrogenase 2 (CKX2) in enhancing grain yield in rice plant. J Biomol Struct Dyn. 2019;38(4):1158-67.

26. Ma X, Feng F, Zhang Y, Eid I, Xu K, et al. A novel rice grain size gene OsSNB was identified by genome-wide association study in natural population. PLoS Genet. 2019;15:e1008191.

27. Nan J, Feng X, Wang C, Zhang X, Wang R, et al. Improving rice grain length through updating the GS3 locus of an elite variety Kongyu 131 . Rice. 2018;11:21.

28. Oladosu Y, Rafii MY, Samuel C, Fatai A, Magaji U, et al. Drought resistance in rice from conventional to molecular breeding: a review. Int J Mol Sci. 2019;20(14):3519.

29. Shakiba E, Edwards JD, Jodari F, Duke SE, Baldo AM, et al. Genetic architecture of cold tolerance in rice (Oryza sativa) determined through high resolution genome-wide analysis. PLoS ONE. 2017;12:e0172133.

30. Suh J-P, Cho Y-C, Won Y-J, Ahn E-K, Baek M-K, et al. Development of resistant gene-pyramided japonica rice for multiple biotic stresses using molecular marker-assisted selection. Plant Breed Biotech. 2015;3:333-45.

31. Miura K, Ashikari M, Matsuoka M. The role of QTLs in the breeding of high-yielding rice. Trends Plant Sci. 2011;16:319-26.

32. Falconer DS, Mackay TFC: Introduction to Quantitative Genetics. 1996;4th edition. Harlow, UK: Longman.

33. Hittalmani S, Huang N, Courtois B, Venuprasad R, Shashidhar HE, et al. Identification of QTL for growth and grain yield-related traits in rice across nine locations of Asia. Theor Appl Genet. 2003;107:679-90.

34. Cho TG, Kang HJ, Lee JS, Lee YT, Lim SJ, et al. Identification of Quantitative Trait Loci in Rice for Yield, Yield Components, and Agronomic Traits across Years and Locations. Crop Sci. 2007;47:2403-17.

35. Wang YH, Li JY. Branching in rice. Curr Opin Plant Biol. 2011;14:94-9.

36. Xing Y, Zhang Q. Genetic and Molecular Bases of Rice Yield. Annu Rev Plant Biol. 2010;61:421-42.

37. Li X, Qian Q, Fu Z, Wang Y, Xiong G, et al. Control of tillering in rice. Nature. 2003;422:618.

38. Koumoto T, Shimada H, Kusano H, She K-C, Iwamoto M, et al. Rice monoculm mutation moc2, which inhibits outgrowth of the second tillers, is ascribed to lack of a fructose-1,6-bisphosphatase. Plant Biotechnol. 2013;30:47-56.

39. Lu K, Wu BW, Wang J, Zhu W, Nie HP, et al. Blocking amino acid transporter OsAAP3 improves grain yield by promoting outgrowth buds and increasing tiller number in rice. Plant Biotechnol J. 2018;16:1710-22.

40. Shinji I, Masahiko M, Tomotsugu A, Kazumitsu O, Itsuro T, et al Junko K. (2005) Suppression of tiller bud activity in tillering dwarf mutants of rice. Plant Cell Physiol. 2005;46:79-86.

41. Lin H, Wang RX, Qian Q, Yan MX, Meng XB, et al. DWARF27, an iron-containing protein required for the biosynthesis of strigolactones, regulates rice tiller bud outgrowth. Plant Cell. 2009;21:1512-25.

42. Liu WZ, Chao W, Fu YP, Hu GC, Si HM, et al. Identification and characterization of HTD2: a novel gene negatively regulating tiller bud outgrowth in rice. Planta. 2009;230:649-58.

43. Yeh SY, Chen HW, Ng CY, Lin CY, Tseng TH, et al. Down regulation of Cytokinin oxidase 2 expression increases tiller number and improves Rice yield. Rice. 2015;8:36.

44. Liu LH, Xie TT, Peng P, Qiu HY, Li XY. Mutations in the MIT3 gene encoding a caroteniod isomerase lead to increased tiller number in rice. Plant Sci. 2017;267:1-10.

45. Xu M, Zhu L, Shou HX, Wu P. A PIN1 family gene, OsPIN1, involved in auxin-dependent adventitious root emergence and Tillering in Rice. Plant Cell Physiol. 2005;46:1674.

46. Lu K, Wu B, Wang J, Zhu W, Nie H, et al. Blocking amino acid transporter OsAAP3 improves grain yield by promoting outgrowth buds and increasing tiller number in rice. Plant Biotechnol J. 2018;16:1710-22.

47. Alam MM, Tanaka T, Nakamura H, Ichikawa H, Kobayashi K, et al. Overexpression of a rice heme activator protein gene (OsHAP2E) confers resistance to pathogens, salinity and drought, and increases photosynthesis and tiller number. Plant Biotechnol J. 2015;13:85-96. 
48. Jiang S, Wang D, Yan S, Liu S, Liu B, et al Kang H, Wang GL. Dissection of the genetic architecture of rice tillering using a genome-wide association study. Rice. 2019;12:43,1-11.

49. Zaw H, Raghavan C, Pocsedio A, Swamy M, Baer ML, et al. Exploring genetic architecture of grain yield and quality traits in a 16-way indica by japonica rice MAGIC global population. Sci Rep. 2019;9:19605.

50. Marathi B, Guleria S, Mohapatra T, Parsad R, Mariappan N, et al. QTL analysis of novel genomic regions associated with yield and yield related traits in new plant type based recombinant inbred lines of rice (Oryza sativa L.). BMC Plant Biol. 2012;12:137.

51. Kumar A, Singh VJ, Krishnan SG, Vinod KK, Bhowmick PK, et al. WA-CMS-based iso-cytoplasmic restorers derived from commercial rice hybrids reveal distinct population structure and genetic divergence towards restorer diversification. 3 Biotech. 2019;9(299)1-15 (2019).

52. Balachandran SM, Sarma NP, Siddiq E. Inheritance of anther culture response in rice. Curr Sci. 1999;77:962-4.

53. Mishra R, Rao GJN, Rao RN, Kaushal P. Development and characterization of elite doubled haploid lines from two indica rice hybrids. Rice Sci. 2015;22(6):290-9.

54. Singh N, Majumder S, Singh ON, Vikram P, Singh A, et al. A large-effect QTL for grain weight in rice on chromosome 10. Aust J Crop Sci. 2015;9(5):372-7.

55. Sruthi K, Divya B, Senguttuvel P, Revathi P, Kemparaju KB, et al. Evaluation of genetic diversity of parental lines for development of heterotic groups in hybrid rice (Oryza sativa L.). J Plant Biochem Biotechnol. 2019. https://doi.org/10.1007/s13562-019-00529-9.

56. Thalapati S, Haritha G, Naga Deepthi N, Prasad Babu A, Surendhar Reddy C, et al. Heterosis and combining ability in rice as influenced by introgressions from wild species Oryza rufipogon including qyld2.1 sub-QTL into the restorer line KMR3. Euphytica. 2015;202:81-95.

57. Mahadevappa M. Rice production in India-relevance of hybrid and transgenic technologies. Indian J Genet Plant Breed. 2004;64:1-4.

58. Wanjari RH, Mandal KG, Ghosh PK, Adhikari T, Rao NH. Rice in India: present status and strategies to boost its production through hybrids. J Sustain Agric. 2006;28:19-39.

59. Han H, Huang B. Application of pollen-derived plants to crop improvement. Int Rev Cytol. 1987;107:293-313.

60. George D, Mallery P SPSS for Windows Step by Step: A Simple Guide and Reference 17.0 Update 10th Edition, Pearson, Boston; 2010.

61. Gravetter F, Wallnau L. Essentials of statistics for the behavioral sciences. 8th Edition, Wadsworth, Belmont, CA; 2014.

62. Konate AK, Zongo A, Kam H, Sanni A, Audebert A. Genetic variability and correlation analysis of rice (Oryza sativa L.) inbred lines based on agromorphological traits. Afr J Agric Res. 2016;11(35):3340-6.

63. Al-Tabbal JA, Al-Fraihat AH. Genetic variation, heritability, phenotypic and genotypic correlation studies for yield and yield components in promising barley genotypes. J Agric Sci. 2012;4(3):193-210.

64. Dutta P, Dutta PN, Borua PK. Morphological trait as selection indices in rice: A statistical view. Univ J Agric Res. 2013;1(3):85-96.

65. Rafii MY, Zakiah MZ, Asfaliza R, Iffah HMD, Latif MA, et al. Grain quality performance and heritability estimation in selected F1 rice genotypes. Sains Malays. 2014;43(1):1-7.

66. Johnson HW, Robinson HF, Comstock RE. Estimation of genetic and environmental variability in soyabeans. Agron J. 1955;47:314-8.

67. Ogunbayo SA, Sie M, Ojo DK, Sanni KA, Akinwale MG, et al. Genetic variation and heritability of yield and related traits in promising rice genotypes (Oryza sativa L). J. Plant Breed. Crop Sci. 2014;6(11):153-9.

68. Nor Aishah H, Harun AR, Mohd Rafii Y, Norain MN, Nur Izzah J. Correlation analysis on agronomic characters in F1 population derived from a cross of Pongsu Seribu 2 and MR 264. Research and Development Seminar 2014; Bangi (Malaysia); 14-16 Oct 2014; INIS-MY-2015016; (2014) INS. 46.

69. Golam F, Yin YH, Masitah A, Afnierna N, Majid NA, et al. Analysis of aroma and yield components of aromatic rice in Malaysian tropical environment. Aust J Crop Sci. 2011;5(11):1318-25.

70. Kohnaki ME, Kiani G, Nematzadeh G. Relationship between morphological traits in rice restorer lines at F3 generation using multivariate analysis. Int J Adv Biol Biomed Res. 2013;1(6):572-7.

71. Thomson MJ, Tai TH, McClung AM, Lai XH, Hinga ME, et al. Mapping quantitative trait loci for yield, yield components and morphological traits in advanced back cross population between Oryza rufipogon and Oryza sativa cultivar Jefferson. Theor Appl Genet. 2003;107:47993.

72. Bernier J, Kumar A, Ramaiah V, Spaner D, Atlin G. A large-effect QTL for grain yield under reproductive-stage drought stress in upland rice. Crop Sci. 2007;47:505-16.

73. Descalsota-Empleo GI, Amparado A, Inabangan-Asilo MA, Tesoroa F, Stangoulisc J, et al. Genetic mapping of QTL for agronomic traits and grain mineral elements in rice. Crop J. 2019;7(4):560-72.

74. Agrama HA, Eizenga GC, Yan W. Association mapping of yield and its components in rice cultivars. Mol Breed. 2007;19:341-56.

75. Li D, Sun C, Fu Y, Li C, Zhu Z, et al. Identification and mapping of genes for improving yield from Chinese common wild rice (O. rufipogon Griff.) using advanced backcross QTL analysis. Chinese Sci Bull. 2002;47(18):1533-7.

Page 17/20 
76. Septiningsih EM, Prasetiyono J, Lubis E, Tai TH, Tjubaryat T, et al. Identification of quantitative trait loci for yield and yield components in an advanced backcross population derived from the Oryza sativa variety IR64 and the wild relative O. rufipogon. Theor Appl Genet. 2003;107:1419-32.

77. Bing Y, Xue WY, Luo LJ, Xing YZ. QTL analysis for flag leaf characteristics and their relationships with yield and yield traits in rice. Acta Genet Sin. 2006;3(9):824-32.

78. Kinoshita N, Kato M, Koyasaki K, Kawashima T, Nishimura T, et al. Identification of quantitative trait loci for rice grain quality and yieldrelated traits in two closely related Oryza sativa L. subsp. japonica cultivars grown near the northernmost limit for rice paddy cultivation. Breed Sci. 2017;67:191-206.

79. Peng JY, Virmani SS. Heterosis in some inter-varietal crosses of rice. Oryza. 1991;28:31-6.

80. Brondani C, Rangel PHN, Brondani RPV, Ferreira ME. QTL mapping and introgression of yield-related traits from Oryza glumaepatula to cultivated rice (Oryza sativa) using microsatellite markers. Theor Appl Genet. 2002;104:1192-203.

81. Zhang ZH, Li P, Wang LX, Hua ZL, Zhuc LH, et al. Genetic dissection of the relationships of biomass production and partitioning with yield and yield related traits in rice. Plant Sci. 2004;167:1-8.

82. Zapata-Arias FJ. Laboratory protocol for anther culture technique in rice. In: Mauszynski M., K.J. Kasha, Forster BP. Doubled haploid production in crop plants, a manual. Kluwer Academic Publishers, 2003;Dordrecht:109-116.

83. Mishra R, Rao GJN, Rao RN. Effect of cold pretreatment and phytohormones on anther culture efficiency of two indica rice (Oryza sativa L.) hybrids: Ajay and Rajalaxmi. JExpBiolAgricSci. 2013;1(2):69-76.

84. Pranathi K, Viraktamath BC, Neeraja CN, Balachandran SM, Prasad ASH, et al. Development and validation of candidate gene-specific markers for the major fertility restorer genes, $R f 4$, and $R f 3$, in rice. Mol.Breed. 2016;36(10):145.

85. Sheeba NK, Viraktamath BC, Sivaramakrishnan S, Gangashetti MG, Khera P, et al. Validation of molecular markers linked to fertility restorer gene (s) for WA-CMS lines of rice. Euphytica. 2009;167:217-27.

86. IRRI. Standard Evaluation System for Rice (SES). Los Banos. Philippines: International Rice Research Institute (IRRI). 4 ed.2002;p. 15-16.

87. Kulkarni SR, Balachandran SM, Ulaganathan K, Balakrishnan D, Praveen M, et al. Molecular mapping of QTLs for yield related traits in recombinant inbred line (RIL) population derived from the popular rice hybrid KRH-2 and their validation through SNP genotyping. SciRep. 2020. 10:13695.https://doi.org/10.1038/s41598-020-70637-3.

88. Dellaporta SL, Jonathan W, James BH. A plant DNA mini preparation: Version II. Plant Mol Biol Rep. 1983;1(4):19-21.

89. Wang J. Inclusive composite interval mapping of quantitative trait genes. Acta Agronomica Sin. 2009;35:239-45.

90. Meng L, Li H, Zhang L, Wang J. QTL IciMapping: Integrated software for genetic linkage map construction and quantitative trait locus mapping in biparental populations. Crop J. 2015;3:269-83.

91. Jaikishan I, Rajendrakumar P, Ramesha M, Viraktamath B, Balachandran S, et al. Prediction of heterosis for grain yield in rice using 'key' informative EST-SSR markers. Plant Breed. 2009;129:108-11.

92. Fiyaz RA, Yadav AK, Krishnan SG, Ellur RK, Bashyal BM, et al. Mapping quantitative trait loci responsible for resistance to Bakanae disease in rice. Rice. 2016;9:3-10.

93. McCouch SR. Gene nomenclature system for rice. Rice.2008;1,7284 (2008).

94. Bai XF, Luo LJ, Yan WH, Kovi MR, Xing YZ. Quantitative trait loci for rice yield-related traits using recombinant inbred lines derived from two diverse cultivars. J Genet. 2011;90(2):209-15.

95. Zhu YJ, Huang DR, Fan YY, Zhang ZH, Ying JZ, et al. Detection of QTLs for Yield Heterosis in Rice Using a RIL Population and Its Testcross Population. Int. J. Genomics. 2016. Article 2587823.

96. Ding X, Li X, Xiong L. Evaluation of near-isogenic lines for drought resistance QTL and fine mapping of a locus affecting flag leaf width, spikelet number, and root volume in rice. Theor Appl Genet. 2011;123:815-26.

97. Kim CK, Chu SH, Park HY, Seo J, Kim B, et al. Identification of heterosis QTLs for yield and yield-related traits in Indica-Japonica recombinant inbred lines of rice (Oryza sativa L.). Plant Breed Biotech. 2017;5(4):371-89.

\section{Figures}



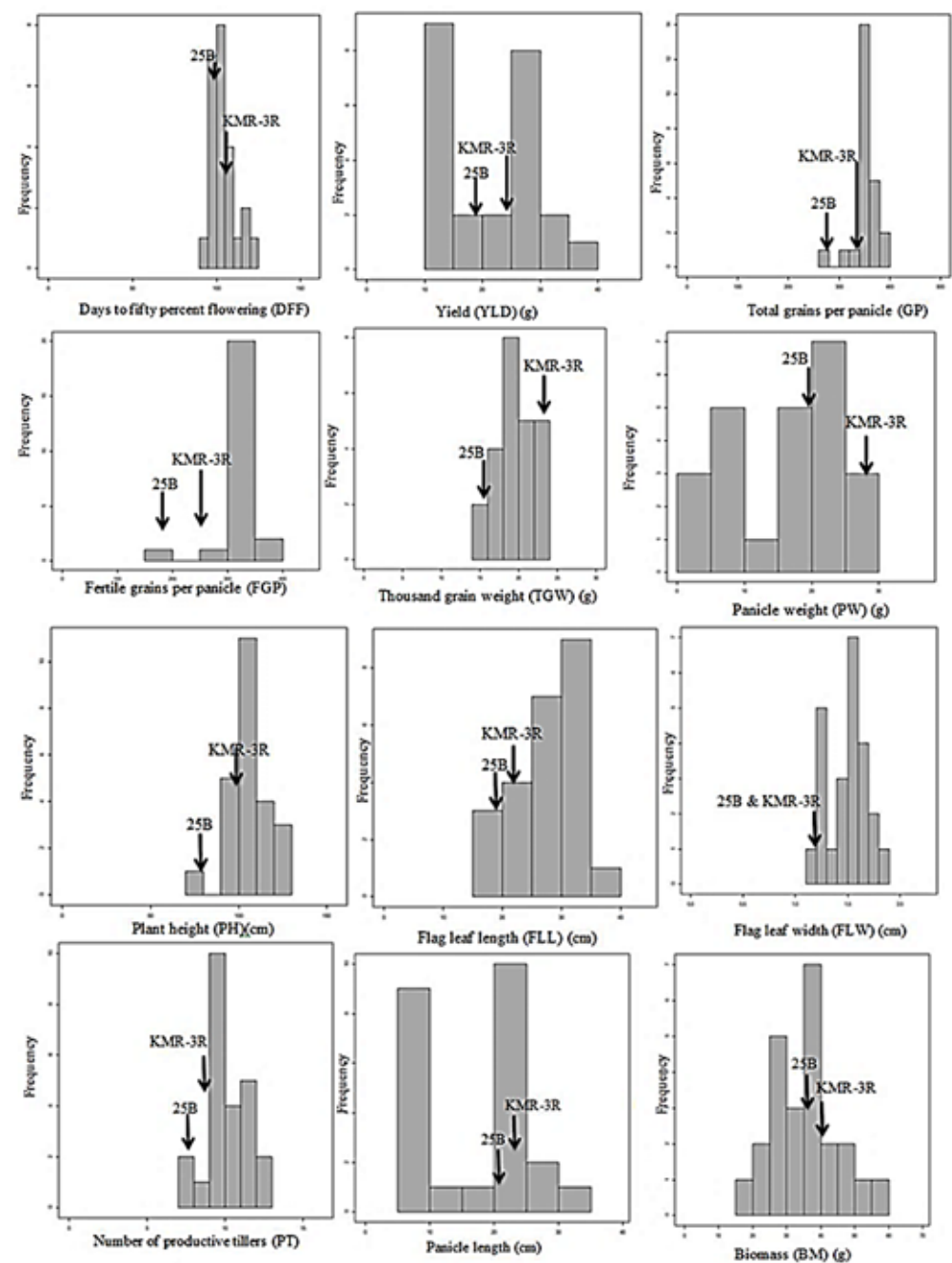

Figure 1

Frequency distribution histograms of the means of 125 individuals of doubled haploid (DH) population for various traits. Mean value of parents, IR58025B and KMR-3R indicated by an arrow. 

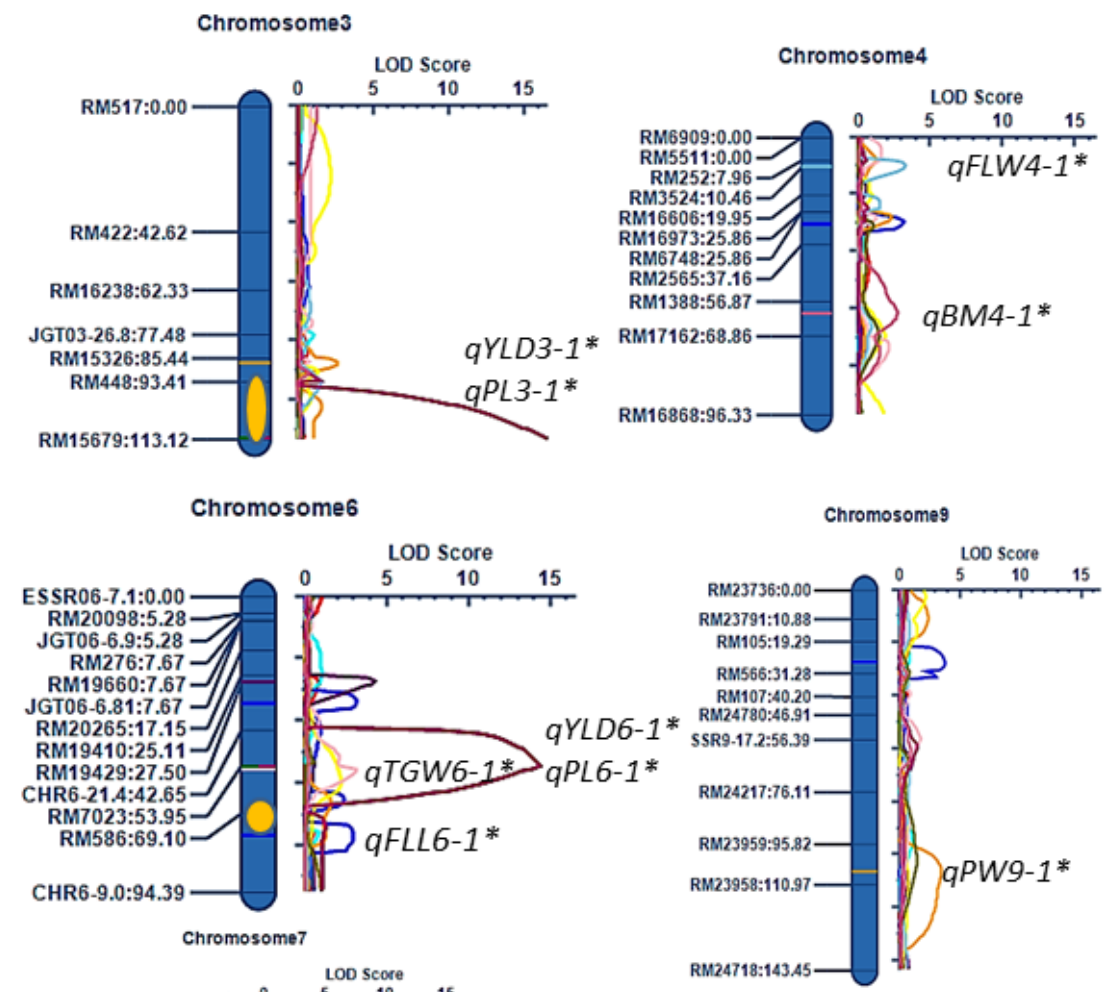

Chromosome9
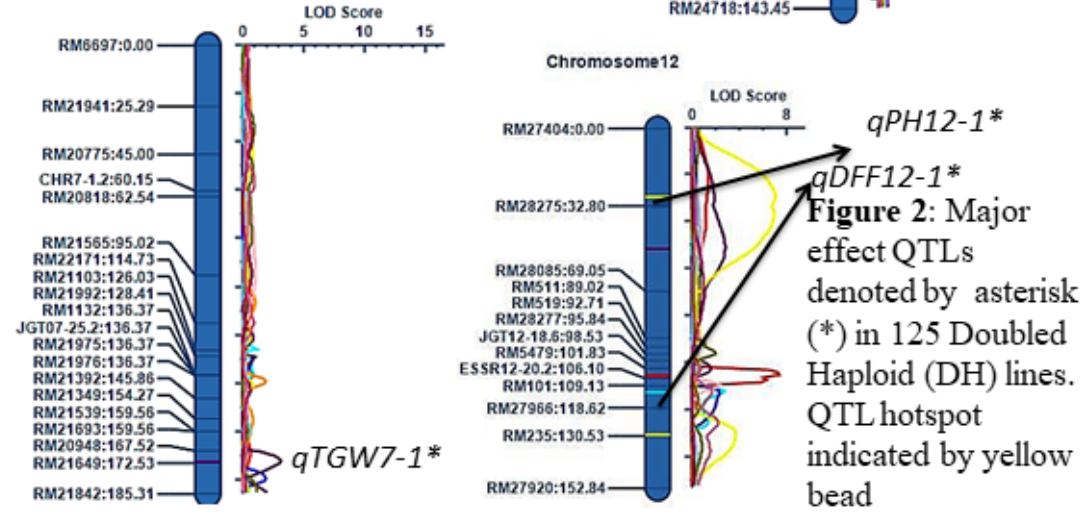

Figure 2

Major effect QTLs denoted by asterisk (*) in 125 Doubled Haploid (DH) lines. QTL hotspot indicated by yellow bead.

\section{Supplementary Files}

This is a list of supplementary files associated with this preprint. Click to download.

- SupplementaryTables22.08.2020.docx

- SupplementaryFigures.pptx 